\title{
ENHANCED MAINTENANCE PROBLEM RECOGNITION TECHNIQUES AND ITS APPLICATION TO PALM OIL MILLS
}

\author{
${ }^{1}$ Basari, A.S.H., ${ }^{2}$ Herman, N.S. and ${ }^{3}$ Asmai, S.A. \\ Faculty of Information and Communication Technology \\ Universiti Teknikal Malaysia Melaka \\ Karung Berkunci 1200, 75450 Ayer Keroh, Melaka, Malaysia \\ Iabdsamad@utem.edu.my \\ 2nsuryana@utem.edu.my \\ 3azirah@utem.edu.my
}

\begin{abstract}
This paper discusses the application of enhanced maintenance problem recognition techniques. The main contribution of this study is the proposed combined techniques, namely snapshot model, failure mode, effect and criticality analysis (FMECA), Pareto analysis, and decision analysis by using information technology (IT). The snapshot model is part of the maintenance modelling technique while FMECA, Pareto analysis, and decision analysis are part of maintenance reliability techniques. Each of the techniques and the proposed combined techniques is explained. The case study used for this enhanced technique was the palm oil mills maintenance problem. The result and possible further enhancement is also discussed.
\end{abstract}

Keywords: Maintenance problem recognition, Snapshot model.

\section{INTRODUCTION}

The maintenance problem recognition is the process of identifying, defining, and diagnosing the maintenance problem. The main purpose of maintenance problem recognition is to avoid tackling the wrong problem. Generally maintenance problem recognition involves (Christer \& Whitelaw, 1983):

(a) identifying the existence and location of the problem-which are recognising the symptoms, seriousness of the problem from the aspect of cost, downtime as well as the size, and the areas of the fault in the plant's machines where the problems are most developed;

(b) determining the problem's causes-the analysis of the problem's causes can be at structural or functional level; consequently depending on the level of causal analysis, different solution strategies may be generated; and 
(c) generating and determining possible solution strategies-having identified the problem and its nature, location, causes, and consequences, only then possible solution strategies could be developed or generated.

However, the data specified above are difficult to be found in any organisation and it is also very tedious to be collected on a dynamic basis if maintenance management information system is supposed to be used. For this reason the use of a survey form for collecting such type of data on a periodic a basis is suggested. A survey form is designed with the collaboration of maintenance engineers and operational research (OR) analysts. The designed survey form is then delivered to the maintenance engineers responsible for the repair of the machines. At each failure or maintenance intervention, the engineer registers the data related to the snapshot model in the survey form. After obtaining a satisfactory sample of the data, OR analyst collects the survey form and starts the analysis process. The results of the analysis, which is either in a graphical or tabular form, is then reported to the maintenance engineers. The results obtained are expected to reveal the true status of the plant under the study.

Despite the usefulness of the snapshot model as one of the important tools for maintenance problem recognition, the implementation of the model in large scale is doubtful. This is mostly due to the scarcity and the reliability of the data related to snapshot model, the problem of analysing the data, and the problem of interpreting the results of the analysis to the users (maintenance engineers). Conducting snapshot modelling on a regular basis possesses problems for maintenance engineers, since each time, they need to recall the OR analysts to help in the analysis and interpretation of the results. Even with the availability of the OR analysts, they need to spend a considerable amount of time in collecting the data, analysing the data, and interpreting the results to the users.

In the current snapshot, model there is an analysis for the major fault / failure types where each component is listed with its number of faults / failures. Assessing the severity of the faults / failures in terms of the frequency of the fault / failure, in this situation, is sometimes misleading. For instance, if a component that has developed the highest number of faults / failures is identified, but does not disrupt the work of the machine completely, and thus incur little cost and downtime, then, ranking such a component as the most critical one is misleading. Even though the ranking is established and proper analysis is conducted, an overall ranking based on all the criteria are not considered. The graphical representation of the analysed data is not analysed 
for which areas are critical or not. This could have no meaning for the users (maintenance engineers) and may lead to wrong decisions being made. Deeper analysis also needs to be considered by look into different angles such as combining the cost with the mode of fault as well as downtime and the criticality of the components based on the probability of failure that could affect whole machines. A so-called decision analysis, a decision-making process technique that could assist the users (maintenance engineers), is also used as part of the snapshot model, which has been proven to assist in making a decision.

\section{THE PROPOSED ENHANCED MAINTENANCE PROBLEM RECOGNITION TECHNIQUES}

The expertise of OR analyst, which is always sought after in the development of a snapshot model, can be reduced with the implementation of a computerised snapshot model. The need for additional type of analysis in the current snapshot analysis can enhance the process of maintenance problem recognition. The proposed approach of automating and augmenting the snapshot model is not aiming to lesser the usefulness of the old snapshot model, but to complement such type of modelling. Enriched techniques that have proven appropriate and possible in combining with the snapshot model could give a more effective, ease of use and practically applicable to the real world maintenance problems.

\section{Elements of Enhancement}

The need to enrich the current snapshot model with additional analysis, and facilitate its development requires the utilisation of the emerging information technology (IT) and failure mode, effect and criticality analysis (FMECA). In theory, IT and FMECA can be utilised to produce an enhanced snapshot model. Once the data collection is done, the analysis process needs further techniques to be used. This is where the techniques called Pareto diagram and decision analysis are introduced. The decision analysis in this study, used an analytic hierarchy process (AHP) method and decision making grid (DMG) by utilising fuzzy logic rule base (FLRB) method, which had been proven appropriate for the industrial maintenance decision analysis process. Figure 1 shows the conceptual merger of the above mentioned techniques into the current snapshot model.

\section{Information Technology}

In the development of the current snapshot model, OR analysts are involved in the design of the data survey form, supervising the collection of the data, analysing the data, and interpreting the results to the engineers. The 
maintenance engineers are not involved in the snapshot model construction. They are only involved in the assistance of OR analysts in the design of the survey form. Computer technology can increase the involvement of maintenance engineers in the development of the snapshot model by allowing the replacement of the survey form with a more general computer form that contains feature checking of the validity and consistency of the data, and can be applied for different machines.

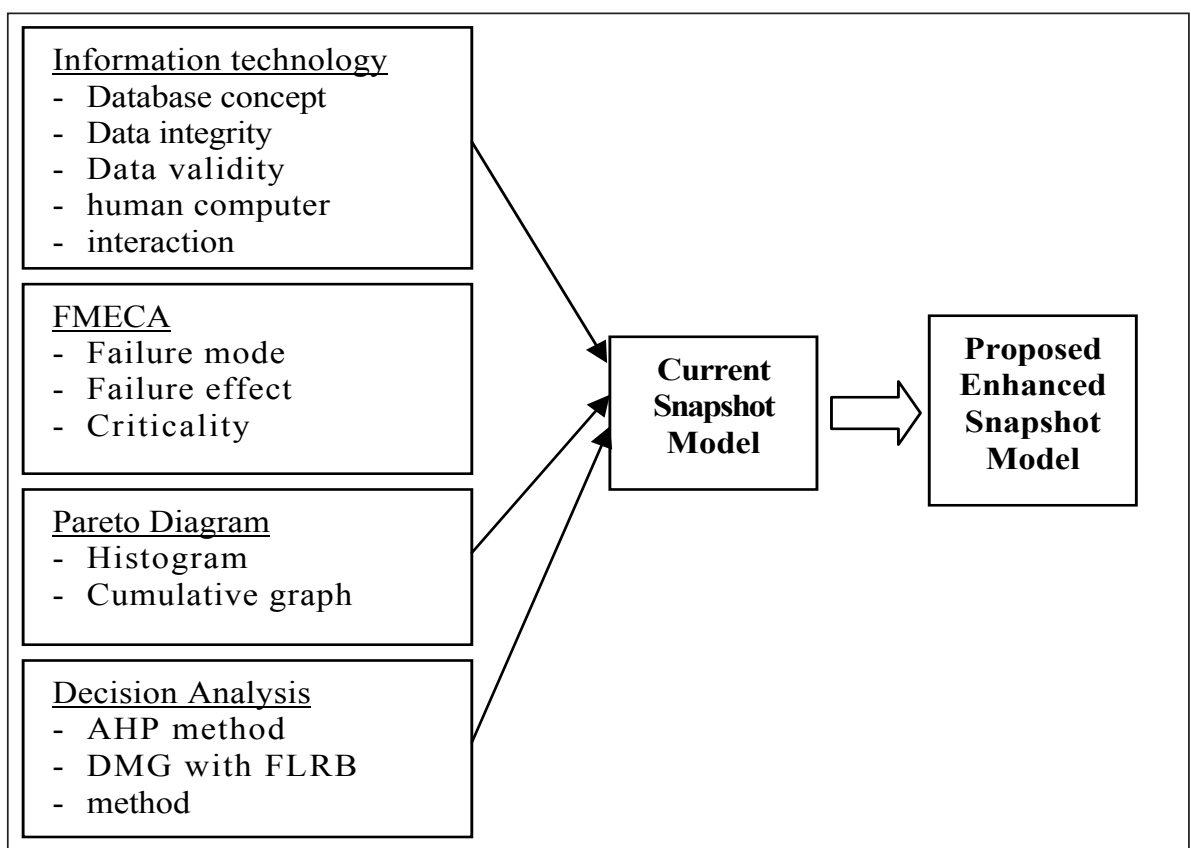

Fig. 1: The Conceptual Merger of FMECA, IT, Pareto Diagram, and Decision Analysis into the Snapshot Model

Computer technology can also permit maintenance engineers to carry out the snapshot analysis with or without assistance of OR analysts.

Failure Mode, Effect, and Criticality Analysis

The failure mode and effect analysis could combined with the snapshot analysis to include:

- major fault areas and their modes-in this kind of analysis, all failures for each component of the machine will be analysed according to their mode;

- failure mode and their cause analysis-this type of analysis provides guidelines and directions which is needed to be performed for specific failure modes; 
- failure modes and their cost analysis-this kind of analysis identifies the consequences of each failure mode in terms of the cost;

- failure modes and their downtime-analysis of such type leads to identifying the failure mode, which frequently disrupts the operation of the machines; and

- failure modes and means of prevention analysis-this kind of analysis identifies the viable means of preventing each type of failure mode.

The term criticality means a relative measure of the consequences of failure mode and its frequency of occurrences. Criticality analysis (CA) is a procedure by which each potential failure mode is ranked according to the combined influence of severity and probability of occurrence. According to Kececioglu (1991), the procedure for obtaining the criticality analysis is as follows;

- the number of failure for each mode will be calculated from the collected data;

- the total number for all the failure of the machine will be calculated;

- the failure mode frequency ratio (FMFR) will be calculated by dividing the number of failure for each mode by the total number of failure for the machines;

- obtain the estimated probability of stopping, $P s$, of the machine if the failure in a given mode should occur;

- obtain the component unreliability $Q$ by subtracting the component's predicted reliability from 1 or 100 (if calculated in percent ages); and

- calculate the Criticality, $C R=(F M F R) \times(P S) \times(Q)$.

By using the above steps, criticality ranking will be conducted for the components of any machine under the study.

\section{Pareto Diagram}

Pareto diagram or analysis is type of data analysis that consists of two main features which are Pareto table and graph. A pareto table shows a tabular form of ranking the worst machines or components, for example ten worst machines are ranked based on their frequency of breakdown. This table could be divided into three sets of low, medium, and high number of frequencies (Labib, 1998). This particular table is already included in the old snapshot model. Pareto graph is a combination of histograms with ranked worst machines and its cumulative percentage. The concept of $80 \%$ of problems/faults coming from $20 \%$ of machines is implemented. This could show that the left side of the graph is more critical than the right side, which means that the more machines go to the right side, the less critical it is. 


\section{Decision Analysis}

Decision analysis is a particular technique that was initiated by Labib (1998) as part of the framework to achieve world class maintenance. Among the established method to implement decision analysis are AHP and DMG based on the FLRB method. The AHP was developed at the Wharton School of Business by Thomas Saaty, (1997). It is a decision support tool, which can lead the decision makers to model a complex problem in a hierarchical structure showing the relationship of the goal, objective (criteria), sub-objectives, and alternatives. Fig. 2 shows the workflow of the AHP process.

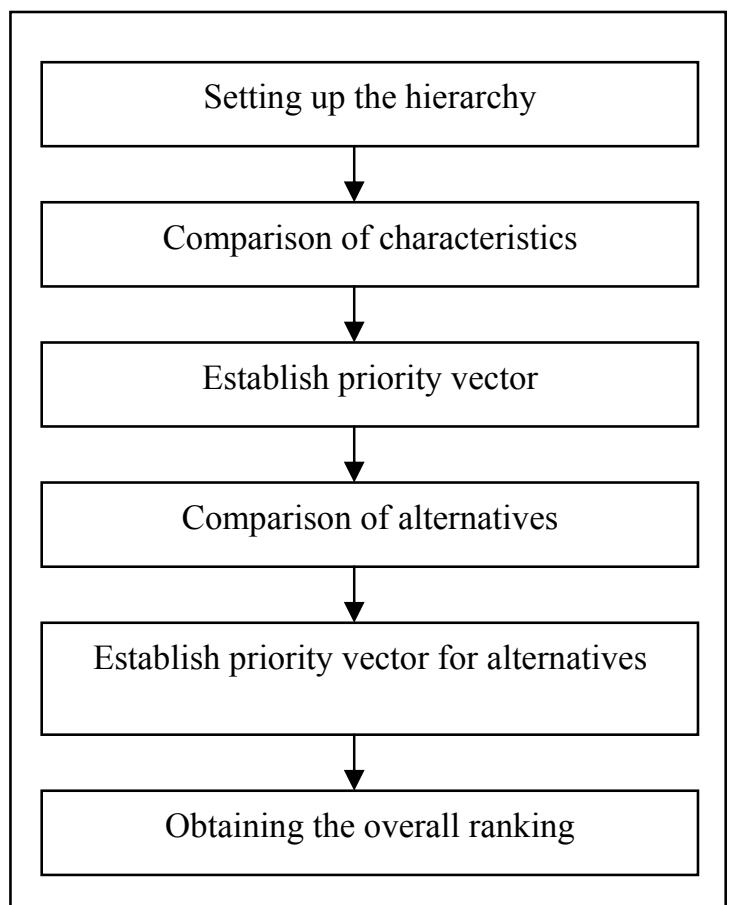

Fig. 2: Analytic Hierarchy Process (AHP) Workflow

There are four major steps to calculate AHP which are;

1. Setting up the hierarchy.

The first step in AHP is to develop a hierarchy by breaking the problem down into its components. This level is also known as the design phase. The three major levels of hierarchy are the goal, objectives, and alternatives. The goal is a statement of the overall objective. The alternative is the feasible way that is available to reach the ultimate goal.

2. Comparison of characteristics and establish priority vector.

Characteristics refer to the objectives or criteria that are located in the 
second level of the hierarchy. In this phase, it is known as the evaluation phase. The decision maker needs to perform a comparison between each objective in a one-to-one $(N \times N)$ matrix form.

Pair wise comparison is used to determine the relative importance of each alternative in terms of each criterion. The pair wise comparison expresses the qualitative answer of a decision maker into some numbers, which is easy to manipulate in the calculation and thus solves the problem of inconsistency in the unit of measurement for each criterion. Pair wise comparisons are quantified by using a scale which is a one-to-one mapping between the set of discrete linguistic choices available to the decision maker and a discrete set of numbers which represent the importance or weight of the previous linguistic choices. Table 1 shows the proposed scale where the scale member set is $\{9$, $8,7,6,5,4,3,2,1,1 / 2,1 / 3,1 / 4,1 / 5,1 / 6,1 / 7,1 / 8,1 / 9\}$.

Table 1: Scale of Relative Importance

\begin{tabular}{|c|c|c|}
\hline $\begin{array}{l}\text { Intensity of } \\
\text { Importance }\end{array}$ & Definition & Explanation \\
\hline 1 & Equal importance & $\begin{array}{l}\text { Two activities contribute equally to } \\
\text { the objective }\end{array}$ \\
\hline 3 & Weak importance of one over another & $\begin{array}{l}\text { Experience and judgement slightly } \\
\text { favour one activity over another }\end{array}$ \\
\hline 5 & Essential or strong importance & $\begin{array}{l}\text { Experience and judgement strongly } \\
\text { favour one activity over another }\end{array}$ \\
\hline 7 & Demonstrated importance & $\begin{array}{l}\text { An activity is strongly favoured } \\
\text { and its dominance demonstrated in } \\
\text { practice }\end{array}$ \\
\hline 9 & Absolute importance & $\begin{array}{l}\text { The evidence favouring one activity } \\
\text { over another is of the highest } \\
\text { possible order of affirmation }\end{array}$ \\
\hline $2,4,6,8$ & $\begin{array}{l}\text { Intermediate values between the two } \\
\text { adjacent judgement }\end{array}$ & When compromise is needed \\
\hline $\begin{array}{l}\text { Reciprocal } \\
\text { of above } \\
\text { (non-zero) }\end{array}$ & $\begin{array}{l}\text { If activity } i \text { has one of the above non- } \\
\text { zero numbers assigned to it when } \\
\text { compared with activity } j \text {, then } j \text { has } \\
\text { the reciprocal value when compared } \\
\text { with } i\end{array}$ & \\
\hline
\end{tabular}

By referring to the above standard scale, a matrix of characteristics (objectives) can be constructed. For consistency, it is necessary to set $\mathrm{a}_{\mathrm{ji}}=1 / \mathrm{a}_{\mathrm{ij}}$ (this states the obvious fact that if objective 1 is slightly more important than objective 3 , than the objective 3 is slightly less important than objective 1 . 
Hence the concept of putting values in a matrix conforming to the following rules;

a. the equal attribute in the matrix is put as 1 (diagonal);

b. the decision maker only needs to fill the upper right triangle of the matrix;

c. for the lower left triangle of the matrix, the value should be the inverse of the corresponding cell in upper right.

3. Comparison of alternatives and establish priority vector for alternatives. The previous steps determined the weight of each objective, so the next step is to determine how well each alternative scores on each objective. The process of calculation is almost similar with the previous step where a pair-wise comparison matrix for each objective is constructed by referring to the scale.

4. Obtaining the overall ranking.

The final step is to obtain a vector of overall scores for each alternative, which can be accomplished by multiplying the weight calculated by each alternative associated to each of the criteria. The first ranked alternative will have the highest weight (highest priority).

One foundation of the AHP is the observation that the human decisionmaking is not always consistent. Consistency suffers when the criteria being compared are subjective in nature. The AHP provides a standard by which the degree of consistency can be measured. If inconsistency exceeds an established threshold, then participants can re-examine their judgements. In the AHP, the pair-wise comparisons in a judgement matrix are considered to be adequately consistent if the corresponding consistency ratio $(C R)$ is less than $10 \%$. Firstly, the columns in the judgement matrix $A$, is multiplied with the resulting vector priority, $w$, and the average of ratio of each element yields an approximation of the maximum eigenvalue, denoted by $1_{\max }$ (an eigenvalue of a square matrix $A$ is a scalar c such that $A w=c w$ holds for some non-zero vector $w)$.

Then the consistency index $(C I)$ value is calculated by using formula $C I=$ $\left(l_{\max }-n\right) /(n-1)$. Next, the consistency ratio $(C R)$ is computed by dividing the $C I$ value by the random index $(R I)$. The $C R$ is the average $C I$ of sets of judgements (from a 1 to 9 scale) for randomly generated reciprocal matrices. The consistency index is shown in Table 2.

For a perfectly consistent decision maker, each ratio in Step 2 equal to $n$. This implies that a perfectly consistent decision maker has $C I=0$. The values 
of $R I$ in Table 2 give the average value of $C I$ if the entries, for example A were chosen at random (subject to the constraints that $\mathrm{a}_{\mathrm{ij}}$ 's must equal 1 , and $\mathrm{a}_{\mathrm{ij}}=1 / \mathrm{a}_{\mathrm{ji}}$ ). If the ratio of $C I$ to $R I$ is sufficiently small, then the decision maker's comparison is probably consistent enough to be useful. Saaty (1990) suggested that if $C I / R I<0.10$, then the degree of consistency is satisfactory, whereas if $C I / R I>0.10$, serious inconsistencies exist and AHP may not yield any meaningful results.

Table 2: Random Index/Random Consistency Index for Different Value of $\mathbf{n}$

\begin{tabular}{cc}
\hline Order of Matrix (n) & $\begin{array}{c}\text { Randomly Generated } \\
\text { Index of Consistency }\end{array}$ \\
\hline 1 & 0 \\
2 & 0 \\
3 & 0.58 \\
4 & 0.90 \\
5 & 1.12 \\
6 & 1.24 \\
7 & 1.32 \\
8 & 1.41 \\
9 & 1.45 \\
10 & 1.49 \\
11 & 1.51 \\
12 & 1.48 \\
13 & 1.56 \\
14 & 1.57 \\
15 & 1.59 \\
\hline
\end{tabular}

The features to enhance the snapshot model are;

i. first level-criteria evaluation: this step needs the decision maker to prioritise his/her preferences on different criteria, such as fault mode, effect, major fault, fault cause, and consequences;

ii. second level sub-criteria evaluation: this step needs the decision maker to prioritise his/her preferences on different sub-criteria, such as number of fault, machine downtime, cost, and criticality; and

iii. third level-Alternatives selection: the machines are ranked according to their weights which are obtained through running an AHP algorithm in an absolute mode and hence a consistency ratio of value zero is assured.

The above mentioned three levels of AHP method steps are complimentary to the three types of analysis provided by snapshot model, which are major fault 
analysis, cause of fault analysis, and consequences of fault analysis. Once the FMECA features called fault mode, effect, and criticality analysis are embedded into the snapshot model, they will also be the added features to the decision analysis process.

The three steps of the fuzzy controller are Fuzzification, Rule evaluation (Inference), and Defuzzification (Cox, 1994). Each of these steps is described below:

1. First step-fuzzification: the first step in the fuzzy controller is the fuzzification process.

The membership function, universe of discourse $U$, is the classifications that are considered in the problem. It is assumed that both frequency and downtime can be classified into High, Medium, and Low. However, each type of input has its specific scale. The decision maker should be able to specify a different scale for each case according to his/her preferences, which are obtained from experience. A trapezoidal function is assumed for simplicity of coding.

2. Second step-rule evaluation: The rule evaluation step can also be explained as an input-output system.

In this step, inputs are expert rules, and fuzzy inputs obtained from the first step (that is, values of $\mathrm{m}$ ), while outputs are fuzzy values of maintenance actions to be carried out. Given two variables of frequency and downtime with each having three subsets of Low, Medium, and High, then one needs at least nine $(3 \times 3)$ rules to describe the model (system). These rules are in the form of $I F \ldots$. . THEN . . statements. Examples of maintenance prescriptions are as follows;

(a) Operate To Failure $(O T F)$,

(b) Fixed Time Maintenance (FTM),

(c) Skill Levels Upgrade (SLU),

(d) Condition Base Monitoring ( $C B M)$, and

(e) Design Out Maintenance (DOM),

A summary of the application of each action, based on the values of Frequency $(F r)$ and Downtime $(D t)$, is given in Table 3. An example of a rule can be "IF downtime is low and frequency is high, THEN improve operators skill". In other words, the repair time is small but occurs frequently which indicates that it is a relatively a trivial type of repair, and hence skills of operators might be updated to repair those kinds of jobs. This rule can be written as follows:

$I F$ frequency is $H I G H$ and downtime is LOW THEN

S. L. $U \quad$ (Rule 7)

Rule 7 is shown in the third row, and first column in Table 3 . The summary of rules is presented in Table 3 . 
Table 3: Summary of Rules for Maintenance Actions

\begin{tabular}{llll}
\hline & Dt (low) & Dt (medium) & Dt (high) \\
\hline Fr (low) & OTF & FTM & CBM \\
$F r$ (medium) & FTM & FTM & FTM \\
$F r$ (high) & SLU & FTM & DOM \\
\hline
\end{tabular}

Notice that if the action is implemented successfully, it is expected that the next state should be a movement toward the northwest direction. In other words, the objective is to transfer the condition of a particular machine (or component) from high to medium and from medium to low for both frequencies and downtime. Once rules are constructed and given the values of the fuzzy inputs ( $m f l, m f m, m f h, m d l, m d m, m d h)$, one can apply the minimum and maximum (AND \& OR Zadeh) inference computations.

3. Third step-defuzzification: this is the final step in the fuzzy controller.

This process is based on the idea of deriving a crisp value from a fuzzy function. These items are arranged according to the degree of difficulty and cost of implementation, starting from the simplest to the more difficult ones. The defuzzification can be performed by deriving the centre of gravity of the area under the curve of the function. Given the cost function of each maintenance action, one can arrange the maintenance actions, the fuzzy output, and the cost scale function. The feedback mechanism offered by the rules grid or DMG of fuzzy logic, as shown in Table 3, in addition to the feedback already offered in AHP in the form of consistency ratio, provides an effective performance.

The above-mentioned FLRB method is used as an enhancement of the snapshot model features called prevention action analysis.

\section{THE CASE STUDY AND RESULT}

This case study demonstrates the application of the above-mentioned techniques and its effect on maintenance performance. This company was used as a pilot study in order to test whether the system meets the user expectation and preference. A number of experts were interviewed and proposals were made during the design and development of the targeted system. Summary of the result is shown throughout this paper. 


\section{Company Background}

The company is a palm oil mill (POM); the main job is processing the Fresh Fruit Bunches (FFB) to become Crude Palm Oil (CPO). In this particular company, there are about 50 major machines or plants. Since the aim of this tool is to assist maintenance engineers establish an appropriate maintenance action, the case study is related to an old palm oil mill, which has been in operation for more than 10 years and uses a conventional method of maintenance problem recognition techniques.

\section{The Result}

Most of the maintenance information found at POM at the time of the study originated from the unstructured daily and lubricant reports. The unstructured daily report only has the date of the report, time of the report, and the description of work. The lubricant report just gives the machine that needs a top-up or change of the lubricant oil, the quantity of oil needed, and a description of work or problem occuring that might cause the need for top-up or change of the lubricant oil.

In the first step, by using the available data, a simple snapshot model was conducted. Data for the period from Jun 2005 to July 2005 was obtained using the existing method of data collection from the company. Summary of the results was presented to the company. Following the presentation of that report, a plan was set for the development of the tool that could provide an analysis to enhance the maintenance problem recognition. The snapshot model was built based on the data collected from POM concerning the most problematic machine namely, Screw Press. Three phases of maintenance data collections were conducted, which were from 1.8.05 to 30.9.05, that aimed to test the applicability of snapshot modelling for POM maintenance problem recognition; data from 1.8.06 to 30.9.06, and 1.11.06 to 31.12.06 were used to compare the result using the old snapshot model and enhanced snapshot model. Samples of the result using the enhanced maintenance problem recognition technique are shown in Table 4 and Table 5. 
Table 4: Snapshot Model Developments and Its Enhancements for Press Machine Dated from 1.8.05 to 30.9.05 at POM

\begin{tabular}{|c|c|c|c|c|c|}
\hline \multirow{3}{*}{$\begin{array}{c}\text { Component Name/Area } \\
\text { of Fault }\end{array}$} & \multirow{2}{*}{\multicolumn{3}{|c|}{ Current Snapshot }} & \multicolumn{2}{|c|}{ Enhanced Snapshot } \\
\hline & & & & \multirow{2}{*}{$\begin{array}{l}\text { FMECA } \\
\text { Criticality } \\
(/ 100)\end{array}$} & \multirow{2}{*}{$\frac{\text { AHP }}{\text { Weight }}$} \\
\hline & $\begin{array}{l}\text { Number of } \\
\text { Breakdowns }\end{array}$ & $\begin{array}{c}\text { Cost } \\
(\mathrm{xRM} 100)\end{array}$ & $\begin{array}{l}\text { Downtime } \\
\text { (Hours) }\end{array}$ & & \\
\hline 29420E Bearing & 19 & 62.88 & 21.50 & 4.75 & $23.94 \%$ \\
\hline $\begin{array}{l}316 \text { Coupling Bolt \& } \\
\text { Nut }\end{array}$ & 20 & 26.10 & 30 & 4.6 & $20.44 \%$ \\
\hline 16004 Bearing & 3 & 9.05 & 31.5 & 4.74 & $13.67 \%$ \\
\hline $120 \times 150 \times 14$ Oil Seal & 15 & 13.50 & 7.5 & 4.05 & $9.28 \%$ \\
\hline 29320E SKF Bearing & 10 & 9.00 & 5 & 4.8 & $7.46 \%$ \\
\hline 3/8" Asbestos Packing & 1 & 0.63 & 1.5 & 4.25 & $6.65 \%$ \\
\hline 22224E Bearing & 6 & 6.30 & 3.5 & 4.76 & $6.31 \%$ \\
\hline 100x130x14 Oil Seal & 7 & 6.30 & 3.5 & 3.99 & $6.21 \%$ \\
\hline 22220E Bearing & 6 & 5.40 & 3 & 4.74 & $5.93 \%$ \\
\hline
\end{tabular}

Table 5: Snapshot Model Developments and Its Enhancements for Press Machine Dated from 1.8.05 to 30.9.05 at POM

\begin{tabular}{lcc}
\hline \multirow{2}{*}{$\begin{array}{c}\text { Component Name/Area of } \\
\text { Fault }\end{array}$} & \multicolumn{2}{c}{ Prevention Action Analysis } \\
\cline { 2 - 3 } & Current Snapshot & Enhanced Snapshot \\
\cline { 2 - 3 } 29420E Bearing & Preventive Maintenance (PM) & Operator Practice (OP) \\
316 Coupling Bolt \& Nut & PM & OP \\
16004 Bearing & Component Redesign (CR) & PM \\
120x150x14 Oil Seal & PM & OP \\
29320E SKF Bearing & PM & OP \\
3/8” Asbestos Packing & OP & OP \\
22224E Bearing & PM & OP \\
100x130x14 Oil Seal & PM & OP \\
22220E Bearing & PM & OP \\
\hline
\end{tabular}




\section{The Discussion of the Result}

Table 4 has shown a comparison between current existing snapshot model and newly enhanced snapshot model. It is obvious that by using the existing snapshot model, users are unable to select the most critical area of fault. This was due to each item, i.e. number of breakdowns, cost, and downtime, being selected differently. For example, in the case of 16004 Bearing, the number of breakdowns is 3, cost is RM9.05, and downtime 31.5 hours, but for 29320E SKF Bearing, the data are 10, RM9.00, and 5 hours respectively. The problem is what items are to be used as an indicator to select the most critical components. In order to solve this particular problem, the enhanced snapshot model is proposed to generalise all the items by using the AHP method. By using this method, all the possible items are considered and the result is ranked according to the calculated weight.

In the case of prevention action analysis, the existing snapshot model decision is based on users' experience and judgment. By referring to the enhanced snapshot model, users' experience and artificial intelligence (AI) techniques, namely FLRB, is used to improve the decision making. This will ensure its accuracy and consistency of the result.

\section{CONCLUSION AND FUTURE WORK}

The importance of the snapshot model as a tool for maintenance problem recognition is recognised. The recent development in computer technology in terms of speed and capacity, coupled with successful research in human computer interaction, plays a considerable role in the development of a successful tool that is capable of constructing a snapshot model. From the result, it showed that the use of enhanced maintenance problem recognition techniques significantly improve the decision context by adding the features of a snapshot model. However, the need to further enhance these tools is essential. One of the recommendations suggestions is by embedding the techniques with in a computerised maintenance management system (CMMS). The quality of data also could be a major issue and it could be resolved by using automated data capturing techniques, such as using a condition monitoring method. This paper has discussed a tool that has been developed by referring to the POM maintenance problem. The need to design and develop the maintenance problem recognition tool for any kind of data, such as in the field of medicine and education, is vital to ensure its robustness. 


\section{REFERENCES}

Christer, A.H., \& Whitelaw, J. (1983). An operational research approach to breakdown maintenance: Problem recognition. Journal of the Operational Research Society, 34, 1041-1052.

Kececioglu, D. (1991). Reliability engineering handbook. Englewood Cliffs, NJ: Prentice Hall.

Labib, A.W. (1998). World class maintenance using computerised maintenance management system. Journal of Quality in Maintenance Engineering, 4, 66-75.

Saaty, T.L. (1997). A scaling method for priorities in hierarchical structures. Journal of Mathematical Psychology, 15, 234 -281.

Saaty, T.L. (1990). How to make a decision: The analytic hierarchy process. European Journal of Operational Research, 48, 9-26.

Cox, E. (1994). The fuzzy systems handbook, USA: AP Professional. 